\title{
Stent implantation into ductus arteriosus: a new alternative of palliative treatment of duct-dependent pulmonary circulation
}

\author{
Mulyadi M Djer, MD; Bambang Madiyono, MD, PhD; Sudigdo Sastroasmoro, MD, PhD; \\ Sukman T Putra, MD; Ismet N Oesman, MD; Najib Advani, MD; Mazeni Alwi, MD, MRCP
}

\begin{abstract}
Background The technical aspects of ductal stenting have been reported, but little is known about the fate of the duct after stent implantation.

Objectives To determine the effects of PDA stenting on the degree of cyanosis, blood oxygen saturation, long-term patency of PDA, and the growth of pulmonary artery and its branches, in patients with duct-dependent pulmonary circulation.

Methods This was a case series study conducted at the Department of Pediatric Cardiology, Institut Jantung Negara (National Heart Institute), Kuala Lumpur, Malaysia from November 1996 to September 2001 on patients with duct-dependent pulmonary circulation who underwent stent implantation. All patients were followed-up until occlusion or block occurred or until the end of follow-up time. The evaluation consisted of clinical manifestations, electrocardiography, chest x-ray, echocardiography, and cardiac catheterization.

Results Stent was successfully implanted in 60 patients (84\%), failed in 7 patients (10\%) and contra indicated in 4 patients $(6 \%)$. There was significant improvement on the degree of cyanosis after stent implantation $(p<0.0001)$. The blood oxygen saturation significantly increased from $74 \%$ (ranged $42-93$; SD 12) before procedure, to $90 \%$ (ranged $62-100 ;$ SD 8 ) afterward $(p<0.0001)$. The mean duration of follow-up time was 14.2 months (ranged 2.5-50.8; SD 13) and the minimal patency of PDA was 11.2 months (ranged 1.147.7; SD 10.7) after implantation. At the end of follow-up, the stents were widely patent in 33 patients (55\%), stenotic in 15 patients (25\%) and spontaneously occluded in 12 patients $(20 \%)$. There was increased growth of pulmonary artery as measured by McGoon ratio from 1.23 (ranged 0.4-2.3; SD 0.4) to 1.81 (ranged 0.7-0.9; SD 0.57), and no distortion of pulmonary artery and its branches found.

Conclusion Stent implantation into ductus arteriosus decreased the degree of cyanosis, increased blood oxygen saturation, maintained long-term patency of ductus arteriosus, and promoted the increased growth of pulmonary artery without distortion of pulmonary artery and its branches [Paediatr Indones 2004;44:30-36].
\end{abstract}

Keywords: stent implantation, duct-dependent pulmonary circulation, $\mathrm{PDA}, \mathrm{McGoon}$ ratio

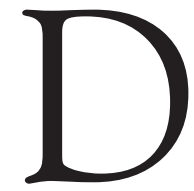

uct-dependent lesion is a congenital heart disease in which the patency of ductus arteriosus is essentially required to maintain either pulmonary circulation or systemic circulation. In duct-dependent pulmonary circulation where survival depends on the presence of the duct, rapid deterioration with increase of cyanosis/hypoxia appears if the duct closes. ${ }^{1}$

So far the surgical creation of an aortopulmonary shunt is still used as a palliative procedure in neonates with duct-dependent pulmonary circulation, and in several institutions has been used as a standard procedure. ${ }^{2}$ However shunt-related complications such as shunt occlusion, shunt stenosis, distortion of pulmonary arteries, pulmonary hypertension and differential growth of the right and left pulmonary arteries, as well as surgical adhesions, increase the complexity and risks of the final definitive surgery. ${ }^{3}$ Currently, stent implantation into ductus arteriosus has been proposed as a non-surgical alternative to aortopul-

From the Department of Child Health, Medical School, University of Indonesia, Jakarta (MMD, BM, SS, STS, INO, NA) and the Department of Pediatric Cardiology, Insitut Jantung Negara, Kuala Lumpur, Malaysia (MA)

Reprint requests to: Mulyadi M Djer, MD, Department of Child Health, Medical School, University of Indonesia, Jakarta, Jalan Salemba 6, Jakarta 10430, Indonesia. Tel. 62-21-3147342, Fax. 3907743 
monary shunt. It offers the potential advantage of eliminating the need for palliative surgery especially in neonatal period, thus reducing the number of operation required, and optimizing the time of definitive surgical correction. ${ }^{3,4}$ We describe our initial experience to asses the safety and efficacy of stent implantation to maintain the patency of ductus arteriosus in duct-dependent lesion.

\section{Methods}

\section{Patient population}

Between November 1996 and September 2002, 71 patients with duct-dependent pulmonary circulation underwent stent implantation into ductus arteriosus as an alternative to surgical palliation on an intention to treat basis. Thirty three patients (47\%) had pulmonary atresia (PA) and intact ventricular septum (IVS), 11 patients had PA and ventricular septal defect (VSD), 6 patients had critical pulmonary stenosis (PS), 6 patients had PA/VSD/ congenitally corrected transposition of great artery (ccTGA), 6 patients had PA/tricuspid atresia (TA), 1 patient had tetralogy of Fallot (ToF), and the remaining 8 patients had other complex congenital heart diseases (CHD) with PA. The hospital committee approved the procedure of stenting into ductus arteriosus. Parental informed consent was obtained in all patients. Before stent implantation performed, all patients were maintained on prostaglandin E1 infusion, ranged from 2 to 46 days (median 15).

Echocardiography was performed to confirm the diagnosis of the underlying heart defects and for the initial evaluation of the anatomy of the duct. At the time of cardiac catheterization, angiography was performed in all patients to demonstrate the morphology of the duct more accurately. The anatomy of ductus arteriosus was delineated by direct selective angiography into the duct using a $4 \mathrm{~F}$ cut pigtail catheter. The anatomy and measurement of maximal lengths and minimal diameters of ductus arteriosus were found using angiography and digital callipers.

\section{Stent type}

A balloon expandable stent was used in this study (Figure 1). Stents were mounted on balloon

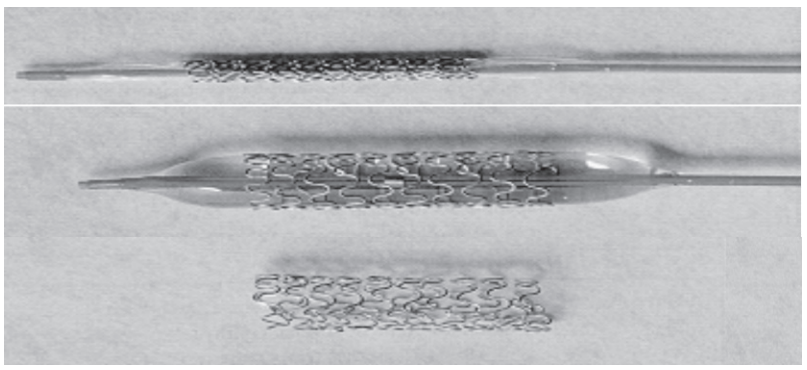

Figure 1. Balloon expandable stent. a. Stent mounted on a balloon angiography catheter, b. During balloon inflated, c. after balloon removed

angiography catheter, positioned across the site of the narrowest site of the ductus arteriosus and implanted by inflating the balloon.

The diameter of the balloon was determined by the expanded diameter of the stent. Stents were mostly made of metals to impart radial strength of the vessel. Stainless steel was used most often as in Palmaz, Bridge, and IntraStents. ${ }^{5}$

\section{Stent implantation}

The procedure of stent implantation into the ductus arteriosus was performed under general anesthesia. After obtaining vascular access, $50 \mathrm{U}$ per $\mathrm{kg}$ heparin was administered intravenously. Cephazolin $30 \mathrm{mg}$ per $\mathrm{kg}$ was administered prior to stent implantation followed by another two doses with interval of 8 hours.

Stent implantation requires various approaches due to the various origins and morphology of the ductus arteriosus and variations in the cardiac defects. In patients with PA/IVS, the approach was either antegradely through the pulmonary artery after RFA/ PTBV was performed to open the atretic valve or retrogradely. While in patients with PA/VSD, the approach was either antegradely through the ascending aorta or retrogradely. For better support during delivery of the stent, a 4F long sheath (Mullin) was used. After entering pulmonary artery (antegradely through PA) or aorta (antegradely through aorta or retrogradely) by using an end hole catheter, a 0.014 coronary wire was introduced via artery route to cross the ductus arteriosus. Over this wire, the delivery system with the coronary stent was advanced into the duct. After the correct position of the stent across the duct was confirmed, the balloon was inflated in order to deploy the stent. 
Repeated angiography of the duct was performed in all patients after stent implantation to confirm the stent position and to exclude persistent stenosis due to incomplete stenting of the duct. In case residual stenosis occurred, an additional stent was implanted to cover the whole duct. After stent implantation, the heparin infusion $25 \mathrm{U} / \mathrm{kg} /$ hour for 48 hours was continued followed by oral aspirin $5 \mathrm{mg} / \mathrm{kg}$ daily.

During follow-up, patients were evaluated by clinical examination, electrocardiography, echocardiography and angiography. Angiography was planned to perform after six months following stent implantation to evaluate stent patency.

\section{Results}

\section{Patients}

The age ranged from 3 days to 34 months (median 26 days) and the weight ranged from 2 to $8 \mathrm{~kg}$ (median 3.3). The stent was successfully implanted in 60 patients $(84 \%)$, failed in 7 patients $(10 \%)$ and contra indicated in 4 patients (6\%). Ninety five percents patients only needed single stent, and the remaining 3 patients (5\%) needed 2 stents each. Stent implantation failed in 7 patients (10\%) because the coronary wire was not able to cross the ductus arteriosus. There was no difference regarding age, weight or sex between patients in the success group and those in the failed group. In the other 4 (6\%), stent implantation was contra indicated because there was stenosis noted in the left pulmonary artery.

Oxygen saturation before stenting with prostaglandin was 74\% (SD 12). Size and length of ductus arteriosus before stenting were $2.3 \mathrm{~mm}(\mathrm{SD} \mathrm{0.7)}$ and $13.0 \mathrm{~mm}$ (SD 4.0) respectively. The McGoon ratio (diameter of the right pulmonary artery at the prebranching point plus that of the left divided by the diameter of the aorta at the diaphragm) based on angiography was 1.23 (SD 0.4).

Most of the patient with PA/IVS group (76\%) had small right ventricle (RV) and the ratio of tricuspid valve annulus and mitral valve annulus was 0.5 (SD 0.2). Ten patients (37\%) had RV coronary fistulae.

Stent implantations were done retrogradely in 28 patients $(47 \%)$, antegradely through pulmonary artery in 23 patients (38\%), antegradely through the ascending aorta in the remaining 9 patients (15\%). For better support during delivery of the stent, a long sheath (Mullin) was used in 28 patients (49.1\%). The diameter and length of the stent were $4.0 \mathrm{~mm}$ (SD 0.3) and $15 \mathrm{~mm}$ (SD 3.3), respectively.

There was significant decrease of the degree of cyanosis before and after stent implantation $(p<0.0001)$. Blood oxygen saturation significantly increased from 74\% (ranged 42-93; SD 12) before the procedure to $90 \%$ (ranged $62-100 ; \mathrm{SD} 8$ ) after $(p<0.0001)$. The diameter of PDA before the procedure was $2.3 \mathrm{~mm}$ (ranged 0.8-4.0; SD 0.7) and increased significantly to $4.3 \mathrm{~mm}$ (ranged $3-7$; SD 0.7) after $(\mathrm{p}<0.0001)$. The diastolic blood pressure significantly decreased from $43 \mathrm{mmHg}$ (ranged 17-78; SD 15) to $33 \mathrm{mmHg}$ (ranged 6-67; SD 12) after the procedure $(\mathrm{p}<0.0001)$.

Mean fluoroscopy time was 33 minutes (SD 18) and mean procedure times were 109 minutes (SD 45). All patients needed ventilation support after procedure with a duration ranged from 1 to 17 day (median 1). Thirty-seven patients (64.9\%) needed ionotropic support post procedure with a duration ranged from 1 to 17 days (median 2). Median of hospital stay was 7 days, ranged from 2 to 45 days.

\section{Mortality}

There were 17 death, 9 hospital death and 8 afterdischarge death. Hospital death occurred at median of 1 day, ranged from 0 to 18 days after procedure. Six of them had PA/IVS with RV hypoplasia (median TV/ MV annulus ratio was 0.4 , ranged from 0.3 to 0.5 ) and RV coronary fistulae. Before stenting, one of them was clinically ill and another 1 patient was a premature baby with birth weight of $1.9 \mathrm{~kg}$. After discharge, death occurred at median of 26 days, ranged from 8 days to 3.7 months. Six of them had PA/IVS with median TV/ MV ratio of 0.5 (range $=0.3-0.6$ ), 3 of them were clinically ill before stenting and 1 patient was a premature baby with birth weight of $1.9 \mathrm{~kg}$.

\section{Complications}

Most of the patients were uneventful during the procedure and the complications occurring during the procedure were only transient mild hypotension and bradycardia. Two patients had serious complications 
and died soon after the procedure. One patient developed endocarditis 1 month after stenting; the stent was removed and BT shunt was done. In another two patients the stent migrated distally and obstructed the LPA; the patient had left BT shunt.

\section{Follow-up}

The mean of follow-up time was 14.2 months (ranged 2.5-50.8; SD 13). At the end of the follow-up time, the stent was widely patent in 33 patient $(55 \%)$, stenotic due to intimal proliferation in 12 patients (20\%), stenotic due to acute angle in 3 patients (5\%) and spontaneously occluded in 12 patients (20\%). Nine out of 12 patients who had stenosis due to intimal proliferation did not need reintervention or surgery. While among patients who had stenosis due to acute angle, 2 patients needed a second stent implantation and 1 patient needed a BT shunt.

There were 12 patients who had spontaneous occlusion at median 7.5 months, ranged from 1.1 and 39.6 months after implantation. Nine patients had PA/ IVS, 2 patients had critical PS and 1 patient had PA/ $\mathrm{VSD} / \mathrm{ccTGA}$. Seven patients did not need reintervention or surgery. The remaining two needed BT shunt and one patient was suitable for Glenn shunt at the time the stent noted closed.

In the group of patients whose stents were stenotic due to intimal proliferation, the mean diameter of stenosis of the inner lumen was 39.6\% (SD 15.1). The stenosis occurred between 4.2 and 47.7 months (median 7) after implantation. Nine of them did not need reintervention nor surgery, while 3 patients needed balloon dilatation. Among patients whose stents were stenotic due to acute angle, 2 of them needed second stent implantation and 1 patient needed BT shunt. At the end of follow-up time, there was significant increased growth of pulmonary arteries as measured by McGoon ratio from 1.23 (SD 0.40) before stenting to 1.81 (SD 0.57) after stenting ( $\mathrm{p}=0.001)$; no distortion of pulmonary artery and its branches was found.

\section{Patency of PDA stenting}

Total spontaneous occlusion was found in 12 patients (20\%) of the entire successful group, which occurred at median of 7.5 months (range 1.1 and 39.6) after PDA stenting. Two of them died and the remaining 10 patients survived. Kaplan-Meier estimated survival showed that the overall patency of the stent in this study was 11.2 months $(95 \%$ CI $7.5 ; 14.9)$ (Figure 2).

\section{Subsequent procedure}

Most of the patients who had either stenosis (9/15) or spontaneous occlusion (7/12) of stent did not need reintervention or surgery. At the end of follow-up time, one patient had biventricular circulation and one patient had Glenn shunt. Kaplan Meier estimated

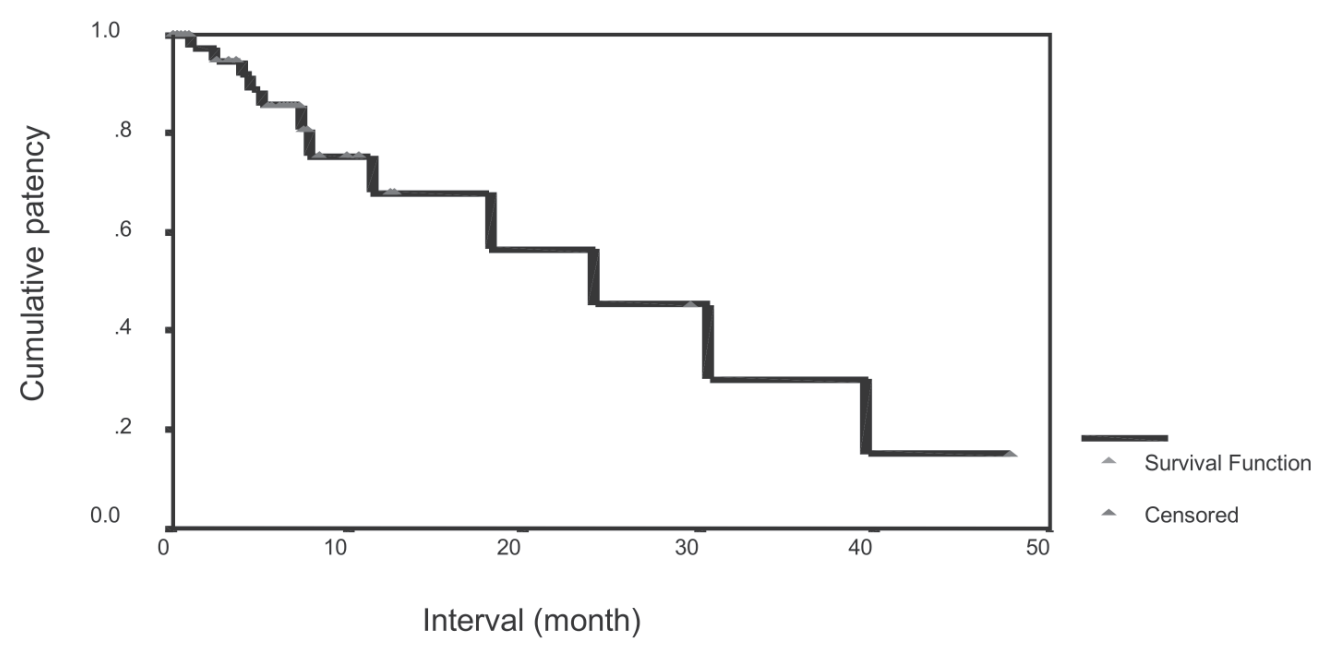

Figure 2. Kaplan-Meier estimated survival of PDA stenting 
survival showed that the percentage of patients who were free from BT shunt was $84 \%$ and the percentage of patients who were free from reintervention was $75 \%$ (Figure 3 and 4) after the initial procedure.

\section{Discussion}

In patients with a duct-dependent pulmonary circulation, patency of ductus arteriosus is essentially required to maintain pulmonary circulation. These patients will have deterioration if ductus arteriosus closes after birth. ${ }^{1}$
Several attempts have been used to preserve the patency of ductus arteriosus. Prostaglandin has been used in the preservation of the duct and stabilized a lot of critically ill infant, but this drug could not be used in long term. ${ }^{6,7}$ Formalin infiltration to preserve ductus arteriosus has been successfully used only to a limited degree and is not used clinically any longer. ${ }^{8}$ Balloon dilatation ${ }^{9,10}$ and thermal balloon dilatation ${ }^{11}$ were investigated in neonatal piglets and lambs, but duct patency was of small caliber and unpredictable. The surgical creation of an aortopulmonary shunt is still used as a palliative procedure in neonates with duct-dependent pulmonary circulation. However

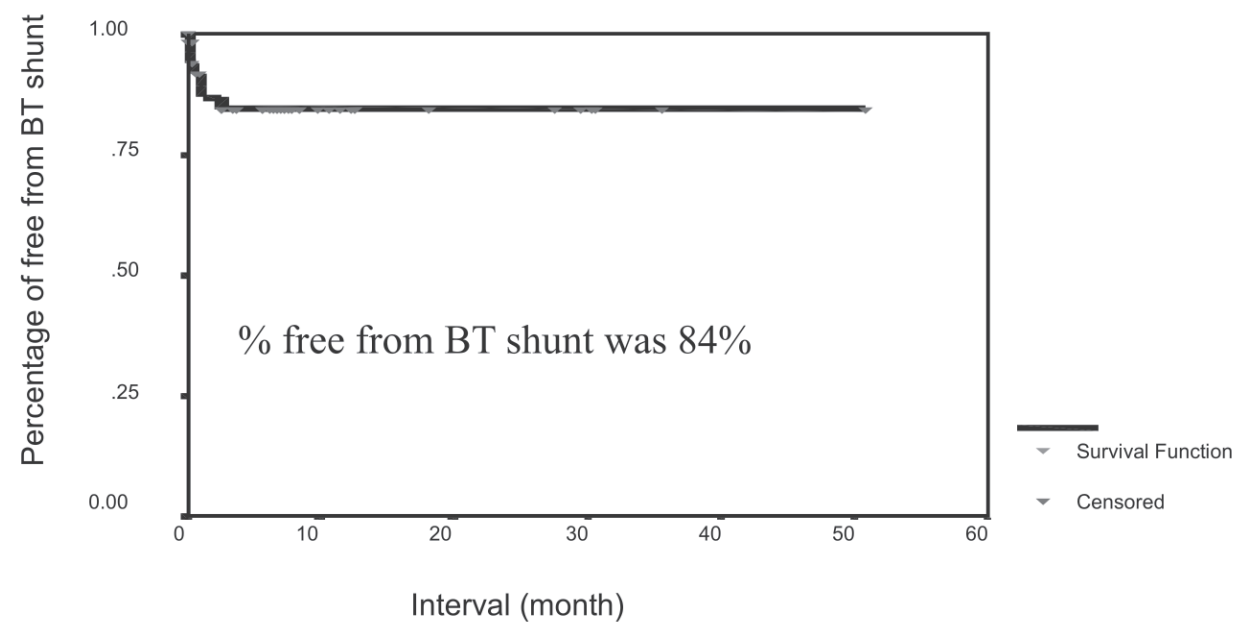

Figure 3. Percentage of patient free from BT shunt after PDA stenting

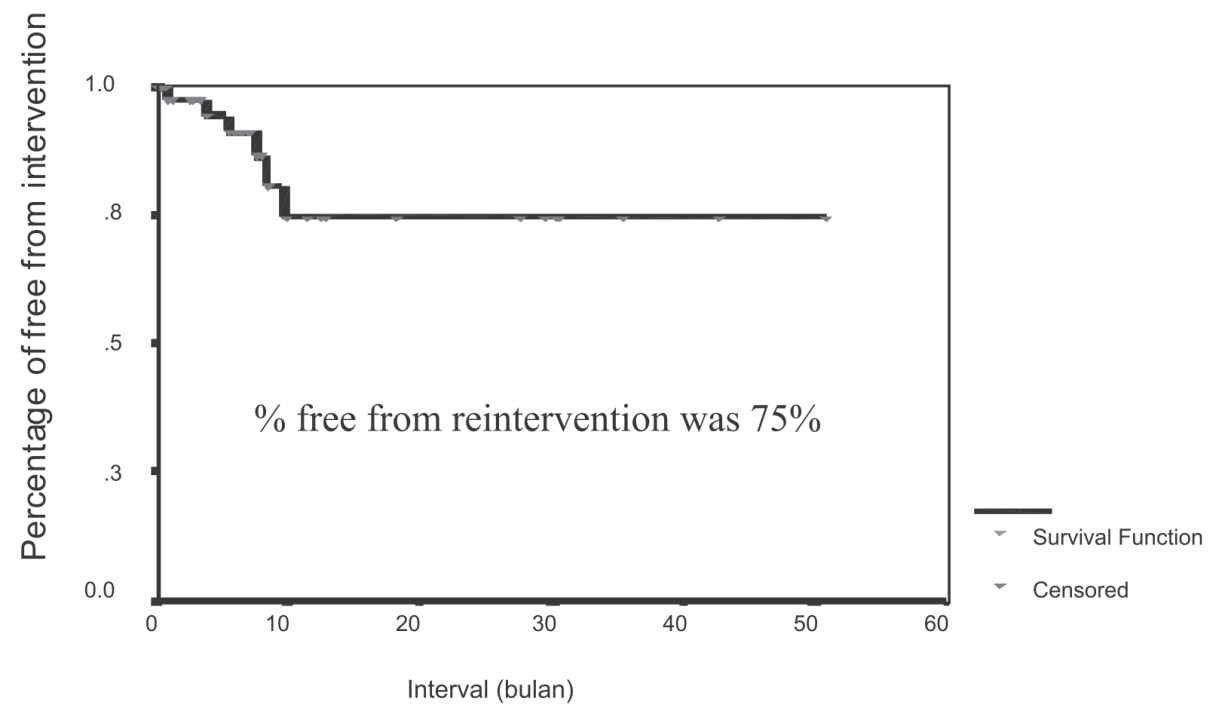

Figure 4. Percentage of patients free from reintervention after PDA stenting 
shunt-related complications such as shunt occlusion, shunt stenosis, distortion of the pulmonary arteries, pulmonary hypertension and differential growth of the right and left pulmonary arteries, as well as surgical adhesions, increase the complexity and risks of the final definitive surgery. ${ }^{2}$ Currently, stent implantation into ductus arteriosus has been proposed as a nonsurgical alternative to aortopulmonary shunt. It offers the potential advantage of eliminating the need for palliative surgery, thus reducing the number of operation required, and optimizing the time of definitive surgical correction. ${ }^{3}$

Encouraging evidence came through the experimental study done by Rosenthal et a ${ }^{12}$ showing that stent implantation into ductus arteriosus maintained its patency up to 21 months and it was superior to balloon dilatation. Schneider ${ }^{3}$ reported his clinical study of stent implantation into ductus arteriosus in 21 patients with duct-dependent circulation. During the follow-up period ranging between 2 months and 2 years (mean 8.7 months), increased growth of the pulmonary arteries was reported in the entire patients and no distortion of the branch of pulmonary arteries was found. Despite the initial enthusiasm, the results of Gibbs et al ${ }^{2}$ showed that stenting was discouraging because of the intimal proliferation requiring reintervention in the majority of patients.

In the group of patients with pulmonary atresia or critical pulmonary stenosis with intact ventricular septum and hypoplastic right ventricle, a staged surgical management approach is often required. In these patients, antegrade flow into the pulmonary circulation from the right ventricle can be established by nonsurgical techniques, such as conventional balloon dilatation of pulmonary valve stenosis or radio frequency valvotomy of the atretic valve. This then allows a second stenting procedure of ductus arteriosus at the same procedure or a few days later to augment the pulmonary blood flow. The additional advantages of this approach is that stent implantation into ductus arteriosus can be performed antegradely via the pulmonary artery. But currently a retrograde approach can be performed safely, so in some cases stent of the ductus arteriosus can be done without RFV. In our institution, formerly we use antegrade approach, but now for patients of PA/IVS with hypoplastic right ventricle we prefer to use retrograde approach to prevent a long procedure and the side effect of radio frequency valvotomy. In retrograde approach by using a long sheath (Mullin) we have advantages to stabilize catheter and wire during crossing the PDA and eliminating additional arterial puncture for aortography during deployment of the stent.

In this procedure, generally, the prostaglandin infusion was stopped 1 day before procedure to avoid duct overdilatation to prevent stent migrating distally. Balloon dilatation to pre dilate the ductus arteriosus was not required in this study. It is recognized that these patients may only require an alternative source of blood supply to the lungs until the right ventricle has adapted or increased in size sufficiently to become capable of supporting the pulmonary circulation alone. Seven from nine patients whose stent closed remained well. By this time the right ventricle had become capable of being the sole contributor to the pulmonary circulation and the occlusion of the stent had no clinical impact.

In another group of heart defects such as PA/ VSD or ToF, the maintainance of the duct was needed until the total correction performed. Besides free from side effects of surgery, in our institution, we aggressively stented the duct because a lot of patients were in waiting list for surgery, so patients did not need to wait too long for BT shunt.

At the end of the follow-up period, there was significant increased growth of pulmonary arteries as measured by McGoon ratio and no distortion of its branches was found. At repeated catheterization, there were five patients who needed reintervention, three needed balloon dilatation of the stent and another two needed a second stent implantation because there was a stenosis at the proximal duct, which was uncovered by a single stent. In contrast to our results, Gibs et $a^{2}$ reported that almost all of his patients needed reintervention.

There were 9 hospital death and 8 after-discharge death. One of 9 patients with early (hospital) death was premorbid ill before underwent the procedure and 1 patient was a premature baby with birth weight of $1.9 \mathrm{~kg}$. Six of 9 patients in the group of early death had PA/IVS with hypoplastic right ventricle (ratio TV/ MV annulus was $0.4 \mathrm{~mm}$, ranged from 0.3 to $0.5 \mathrm{~mm}$ ) and most of them had RV coronary fistulae. Amongst hospital death, four out of 9 deaths were caused by myocardial ischemia and the remaining 2 due to sepsis and subsequently 1 patient died due to post epi- 
cardial pace maker implantation, acute renal failure and RV failure. According to our experience, the outcome of PA/IVS with hypoplastic right ventricle was poor. ${ }^{13}$ In the early experience stent implantation was done antegradely by opening atretic pulmonary valve using RFA/PTBV. Most patients who died in this study were PA/IVS patients, who had RFA/PTBV prior to PDA stenting. Currently, PA/IVS stent implantation can be performed retrogradely without opening atretic pulmonary valve.

In the group of after-discharge deaths, six from 8 patients also had PA/IVS, and the remaining 1 patient had critical PS, and in 1 had TA/PA. Cause of death was sepsis in 3 patients, after BT shunt in 3 patients. One patient was reported dead at home 1 month after procedure without known exact cause of death.

We concluded that in patients with duct dependent pulmonary circulation stent implantation into ductus arteriosus decreased the degree of cyanosis, increased blood oxygen saturation, maintained long-term patency of ductus arteriosus and promoted increased growth of pulmonary artery without distortion of pulmonary artery and its branches. Even though in this study some of the patients had spontaneous occlusion, when it occurred, the right ventricle size had increased, so the circulation was no longer duct-dependent because the right ventricular had become capable of being the sole contributor to the pulmonary circulation. Thus intervention therapy might replace surgical therapy, thereby avoid long-term prostaglandin infusion in patients with complex congenital heart diseases and duct-dependent pulmonary circulation.

\section{Limitations}

This procedure now is still on going in our institution. Shorter follow-up in our study made the patency of the stent was not long enough. In dead patients, we had not done autopsy routinely, so the exact cause of death and the histology of their duct could not be determined.

\section{References}

1. Wilkinson JL. Initial management and referral for surgical interventional of neonates with critical congenital heart disease. Indones J Pediatr Cardiol 2002;1:4-6.
2. Gibbs JL, Uzun O, Blackburn MEC, Wren C, Hamilton JRL, Watterson KG. Fate of the stented arterial duct. Circulation 1999;99:2621-5.

3. Schneider M, Zartner P, Sidiropoulos, Konetz W, Hausdorf G. Stent implantation of the duct in newborns with duct-dependent circulation. Eur Heart J 1998;19:1401-9.

4. Ruiz CE, Gamra H, Zhang HP, Garcia EJ, Boucek MM. Brief report: stenting of the ductus arteriosus as a bridge to cardiac transplantation in infants with the hypoplastic left heart syndrome. New Engl J Med 1993;328:1605-8.

5. Rao PS. Interventional pediatric cardiology: state of art and future directions. Pediatr Cardiol 1998;19:107-24.

6. Freed MD, Heymann MA, Lewis AB, Roehl SL, Kensey RC. Prostaglandin E1 in infants with ductus arteriosus-dependent congenital heart disease. Circulation 1981;64:899-905.

7. Lewis AB, Freed MD, Heyman MA, Roehl SL, Kensey RC. Side effect of prostaglandin E1 in infants with critical congenital heart disease. Circulation 1981;64:893-8.

8. Rudolph AM, Heyman MA, Fishman N, Lakier JB. Formalin infiltration of the ductus arteriosus. A method for palliation of infants with selected congenital cardiac lesions. N England J Med 1975;292:1263-8.

9. Lund G, Rysavy J, Cragg A, Salomonowitz E, Vlodaver Z, Zuniga WC, et al. Long-term patency of the ductus arteriosus after balloon dilatation: an experimental study. Circulation 1984;69:772-4.

10. Lund G, Cragg A, Rysavy J, Castenada F, Salomonowitz E, Vlodaver Z, et al. Patency of the ductus arteriosus after balloon dilatation: an experimental study. Circulation 1983;68:621-7.

11. Abrams SE, Walsh KP, Diamond MJ, Clarkson MJ, Sibbons P. Radio frequency thermal angioplasty maintains arterial duct patency. An experimental study. Circulation 1994;90:442-8.

12. Rosenthal E, Qureshi SA, Tabatabaie AS, Persaud D, Kakadekar AP, Baker EJ, et al. Medium-term results of experimental stent implantation into the ductus arteriosus. Am Heart J 1996;132:657-63.

13. Alwi M, Kandhavello G, Bilkis AA, Lim MK, Samion $\mathrm{H}$, Latiff HA, et al. Pulmonary atresia with intact ventricular septum percutaneous radio frequencyassisted valvotomy versus surgical valvotomy and Blalock Taussig shunt. J Am Coll Cardiol 2000;35:468-76. 